\title{
CONTROLLER BENCHMARKING BASED ON ECONOMIC BENEFITS
}

\author{
Hao Xia*, Pawel Majecki*, Andrzej Ordys*, Mike Grimble* \\ * Industrial Control Center, University of Strathclyde, Glasgow, G1 1XW, U.K. \\ Email: hao.xia@strath.ac.ukＴele: 0044-141-5484200
}

Keywords: benchmarking, constraints, constrained optimization

\begin{abstract}
Benchmarking is a tool for improvement and should be considered as a benefit-driven activity. The ultimate goal of benchmarking is performance improvement. One of major difficulties in the benchmarking of MIMO systems comes from the shear number of control loops contained in a plant. By directly focusing on the improvement of plant's economic performance, we present a controller benchmarking framework based on the existing multi-level control hierarchy. At each level of the hierarchy, a constrained optimization which reflects the economic objective is performed. The active constraints recorded in the optimization process are treated as the goal of improvement in the lower levels. Consequently the constraints in each layer which limit the performance of the plant are isolated. The retuning decision is made based on the result of optimization of an economic metrics. In this manner, the benchmarking problem becomes not only manageable but also meaningful.
\end{abstract}

\section{Introduction}

Benchmarking is first and foremost a tool for improvement, achieved through comparison with the best within the field. The purpose of a benchmarking study is not only comparing for the sake of evaluation, but also looking for the best ways to achieve improvements. Nowadays, manufacturing and process industries are competing globally. A few percentage increase in the profitability will have a huge impact on the success of a company. This recent focus on controller benchmarking is motivated by the importance that control systems have in enabling companies to achieve goals related to quality, safety and asset utilization.

Controller benchmarking has been an active research area for the recent ten years $[2,8,11]$. This recent resurgence of interest in control loop performance assessment is due to the work of Harris [7]. In this paper, Harris proposed the use of closed-loop data to evaluate and diagnose controller performance using minimum variance control (MVC) as a benchmarking metric. Since the minimum variance controller achieves the minimum output variance among all linear controllers, its performance was used as a lower bound to assess the performance of single loop controllers. This idea was further developed in $[2,3]$.

Most of available results are for the benchmarking of SISO systems. In comparison with SISO benchmarking, the effort needed to benchmark a MIMO system grows substantially with the size of the plant. There are two major problems associated with benchmarking MIMO systems. The first one is the coupling between loops of the system. Since the interaction between loops is inherent in the process, it has to be tackled directly. The other problem is the total number of loops contained in such a system. In a typical continuous process plant, there are hundreds to thousands of control loops. Identifying the loops which have the biggest impact on the economic performance of the plant and subsequently improve its performance will definitely improve the performance of the plant.

The rest of the paper is organized as follows: in section two, we present a brief introduction to the controller benchmarking practice. The desirable properties of benchmarking metrics are also discussed. A benchmarking framework based on economic returns is presented in section three. In section four, we perform a benchmarking exercise on a crude oil separation system. The paper is concluded in section five.

\section{Controller benchmarking}

When a plant was commissioned, the controllers were designed to perform the required specifications. However, as time goes by, the changes in the plant dynamics, disturbance characteristics, performance requirements, wear and tear of the controllers, improper retuning (detuning) of controllers; all these lead to the deterioration of the process's performance. Estimates of the percentage of industrial process controllers with performance problems are surprisingly high [9] - various studies indicate that anywhere from $66 \%$ to $80 \%$ of controllers are not performing as well as they should [10]. These controllers can have a significant detrimental effect on 
plant profitability, both in terms of increased product variance and increased reaction times.

In order to tackle the above mentioned problem, controller benchmarking is employed to identify the under performing loops and suggest the best way of improvement. The idea of controller benchmarking stems from the practice of business benchmarking which was initiated in the early seventies by Xerox. Its focus is on the improvement of economic return of the critical processes. One distinctive feature of controller benchmarking is that special attentions are paid to the computation of meaningful benchmarks and the cost and benefits of improvements. In the case of controller benchmarking, we are not only interested in maintaining the current performance, but also interested in improving the performance to the optimal level. Designing a suitable controller ensures the success of controller benchmarking practice. However, controller benchmarking is more than a controller redesign practice. The decision for controller redesign is only justified by the results from the systematic analysis of the current performance.

\subsection{The selection of benchmarking metrics}

In order to analyze the performance of current system, a set of metrics is needed. There are different kinds of measurements which can indicate different aspects of controller performance. However, not all these measurements are suitable for the benchmarking purpose. Ideally a controller assessment metric should have the following attributes:

1. Controller oriented.

- Independent of disturbance or set-point spectrum.

- Sensitive to detuning and process model mismatch or equipment problems.

2. Easily obtainable

- Does not require plant tests.

- Able to be automated.

- Requires minimum specification of process dynamics.

3. Objective and accurate

- The confidence interval of the metric should be provided or the accuracy can be tested by plant data.

- Absolute or non-arbitrary measure.

4. Improvement indicator

- Realistic and achievable under the physical constraints.

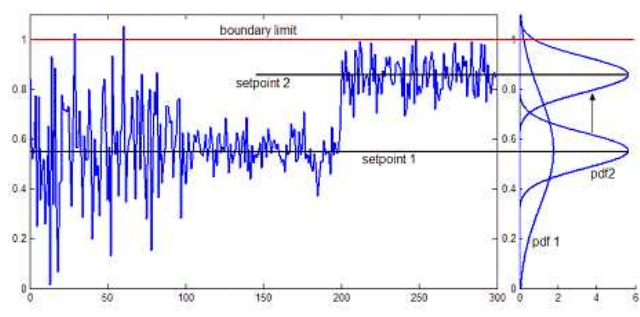

Figure 1: Increasing economic performance by the reduction of variance

- Indicative of why the controller is performing poorly.

- Measures the improvement in profit due to the controller.

It is a stringent requirement for any benchmarking metrics to possess all these attributes. But as a guideline, any benchmarking metrics to be used should possess as many above mentioned attributes as possible. These guidelines can also be used as a reference when selecting a benchmarking metric.

\subsection{MVC benchmark}

In this subsection, we use MVC benchmark to show the points raised above. The performance of a process control loop can often be assessed in terms of the variance of the controlled variable, since it indicates the consistency of the product quality. A lower variance also implies the possibility to move the set-point to the limiting boundary (see figure 1), resulting in increased economic reward. This is a major reason why so many efforts are focused on MVC benchmarking activities.

Assume that the system is a minimum phase system and its dynamics can be represented as:

$$
y(i)=z^{-k} \frac{B\left(z^{-1}\right)}{A\left(z^{-1}\right)} u(i)+\frac{C\left(z^{-1}\right)}{A\left(z^{-1}\right)} d(i)
$$

where $k$ is the process time delay. Under the assumption of zero set-point, the cost function to be minimized is the expected value of output variance:

$$
J=E\left\{y^{2}(i+k)\right\}
$$

The minimum variance controller was derived in [1]:

$$
C_{m v}\left(z^{-1}\right)=\frac{G\left(z^{-1}\right)}{B\left(z^{-1}\right) F\left(z^{-1}\right)}
$$

while $G\left(z^{-1}\right)$ and $F\left(z^{-1}\right)$ are the solution of the following Diophantine equation:

$$
\frac{C}{A}=F+z^{-k} \frac{G}{A}
$$


with $\operatorname{deg} F=k-1$ and $\operatorname{deg} G=\max (\operatorname{deg} A-$ $1, \operatorname{deg} C-k)$.

While the MV controller requires the specification of process and disturbance transfer functions, Desborough and Harris [2] showed that equation (2) can be determined using only closed-loop data and the information of process time delay. The MVC benchmark has many desirable theoretical and practical properties. For example it is data-driven and can be simply automated. Theoretically, this estimation also represents a lower bound on what can be achieved by any linear controller.

But it may fail at requirement (1) - it gives varying results depending on the actual disturbance that enters the process and is not always sensitive to process model mismatch. It also fails at requirement(4) - since the benchmark is usually not achievable. Nevertheless, MVC benchmarking is widely used due to its simplicity and the minimum requirement for $a$ priori knowledge about the system.

Controller benchmarking based on MVC metric also illustrates the major difference between controller benchmarking and controller design. In the case of designing a minimum variance controller, a full model of the plant is required. For controller benchmarking, there is no such requirement in the analyzing phase. The accurate model is only needed after the decision has been made that a retuning of the controller is really needed.

\section{Controller benchmarking based on economic metrics}

As discussed in the previous section, the MVC benchmark can be derived directly from the closed loop data. Based on this, the performance gap between the current controller and the 'ideal' controller can be obtained. Although it is desirable to minimize the output variance, MVC benchmark alone does not indicate any economic reward due to the reduction of output variance. Based on a MVC benchmark alone, the re-tuning of controller is not justified.

In a typical continuous process plant, there are hundreds to thousands of control loops. It is impossible and impractical to benchmark each of them. The priority should be put on finding a set of control loops which could generate the biggest economic returns by retuning. We propose to use economic metrics directly to measure the process performance. From this analysis, we are trying to find which part of the controller limits the process performance. The idea is similar to the so called debottlenecking which was widely used in business benchmarking practice.

\subsection{Loop prioritization based on economic met- rics}

Although a plant may consist of thousands of control loops, it can always be represented in multi-level hierarchy based on control functions, see e.g. figure 2,

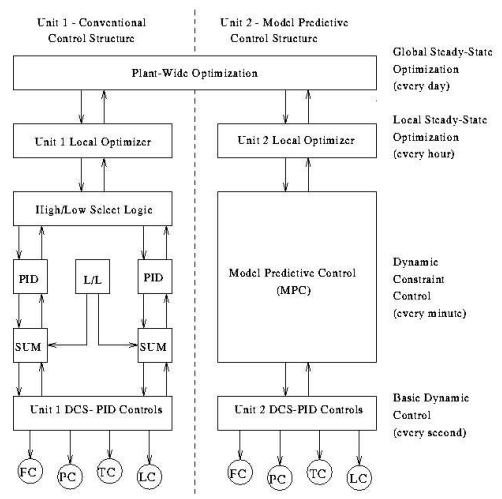

Figure 2: A outline of control hierarchy

Figure 2 shows a conventional control structure on the left for Unit 1 and a model predictive control structure on the right for Unit 2. At the top of the structure a plant-wide optimizer determines optimal steady-state settings for each unit in the plant. These may be sent to local optimizers at each unit which run more frequently or consider a more detailed unit model than is possible at the plant-wide level. The unit optimizers compute optimal economic steadystates and pass these to the dynamic constraint control systems for implementation. The dynamic constraint control must move the plant from one constrained steady state to another while minimizing constraint violations along the way. In the conventional structure this is accomplished by using a combination of PID algorithms, Lead-Lag (L/L) blocks and High/Low select logic. In the MPC methodology this combination of blocks is replaced by a single MPC controller. At the bottom of the hierarchy are the basic dynamic control loops used for tracking and regulation.

At the top two levels of the control hierarchy, static/dynamic optimization is involved to optimize some pre-specified economic metrics. If MPC controller was used in the third level, then another constrained dynamic optimization would also be required. An important characteristic of these optimizations is that they are all constrained optimization problems. Most of the constraints come from the lower levels of the hierarchy. For example, the constraints of MPC controllers come from the regulatory level, while the limitations of MPC controller 
has to be taken into consideration when a high level economic optimization is performed.

A typical static optimization problem can be written as:

$$
\begin{aligned}
& \min _{y_{s}, u} J^{*}\left(y_{s}, u\right) \\
& \text { s.t. } y=f_{s}(u), u_{\min } \leq u \leq u_{\max } \\
& y_{\min } \leq y_{s} \leq y_{\max }, g\left(y_{s}, u\right) \leq 0
\end{aligned}
$$

The model used is a static model and $J^{*}\left(y_{s}, u\right)$ is a predefined economic metric. The constraints on the system input and output come from the lower level of the hierarchy. Depending on the type of $J^{*}\left(y_{s}, u\right)$, in many cases, the optimal solution for (4) is obtained on the boundary of the constraints, and these constraints are called 'active constraints', see figure 3 .

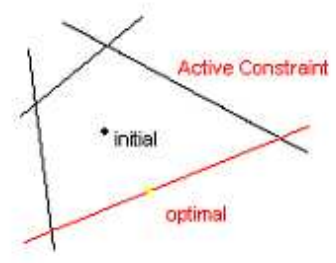

Figure 3: An illustrative solution for a static optimization

The constraints in a plant can be classified into two types:

- Hard constraints - the ones that can not be changed by re-tuning the controller.

- Soft constraints - the ones that can be changed by controller tuning.

From figure 3 , it is not hard to find that the active constraint limits the achievable value for $J^{*}\left(y_{s}, u\right)$. If the active constraint is a soft one, then by pushing this constraint outward, a better economic performance can be expected. It is natural to ask the following questions:

\section{Can these constraints be pushed further?}

2. Do we need to re-tune the controller?

The first question can be answered by using control loop benchmarking techniques such as MVC benchmarking . This information provides guideline on which part of the plant should be investigated. The second question is more involved, and can be con- verted into another optimization problem:

$$
\begin{aligned}
& \max _{C} \Delta J^{*}(y, u)-J_{c}(y) \\
& \text { s.t. } \Delta J^{*}(y, u)-J_{c}(y) \geq 0 \\
& y(k+1)=f(y(k), y(k-1), \ldots, u(k), u(k-1), \ldots) \\
& u_{\text {min }} \leq u \leq u_{\max }, y_{\text {min }}^{\prime} \leq y \leq y_{\text {max }}^{\prime}, \\
& g(y, u) \leq 0
\end{aligned}
$$

where $\Delta J^{*}(y, u)$ is the economic benefit obtained from pushing constraints outward through controller retuning, while $J_{c}(y)$ is the cost involved in retuning the controller. Correct definition of $J_{c}(y)$ is a difficult problem, which should be defined by discussions with the industrial partners. Another effective way of defining $J_{c}(y)$ is through economic auditing.

Equation (5) is actually a controller benchmarking metric. It provides a link between economic benefit and the classical benchmarking techniques such as MVC benchmarking. By focusing on the active constraints obtained from (4), we only need to benchmark the subsystems (or control loops) related to them. The number of control loops that need to be investigated can be significantly reduced. The benchmarking problem becomes not only manageable but also meaningful. Furthermore, from the solution of (5), the loop prioritization is achieved, the retuning of the identified loops will definitely bring the maximized economic benefit.

In (5), the term $\Delta J^{*}(y, u)$ can be obtained easily from (4) by relaxing the constraints. It can be easily computed by using the economic optimizer already installed in the plant. The term $J_{c}(y)$ can be hard to compute, which makes (5) difficult to solve. However, if we impose a restriction that the controller to be re-tuned keeps the original structure, then applying the optimal controller simply means updating the controller parameters. In this case, $J_{c}(y)$ can be set to zero. The optimization problem would be greatly simplified. Some recent results on restrictedstructure controller design can be found in $[4,5]$. In summary, the benchmarking process based on economic metrics is illustrated in figure.4.

\section{Controller benchmarking of a oil sep- aration system}

\subsection{Process description}

The separation system is designed to process reservoir fluids [6]. Crude oil, flash gas and water are separated in a separation train comprising three separation vessels (representative of the three-stage separation process) and then passed downstream to other process units. The inflow of reservoir fluids into this separation train can be described as oscillatory with high amplitude and can be regarded as a sinusoidal 


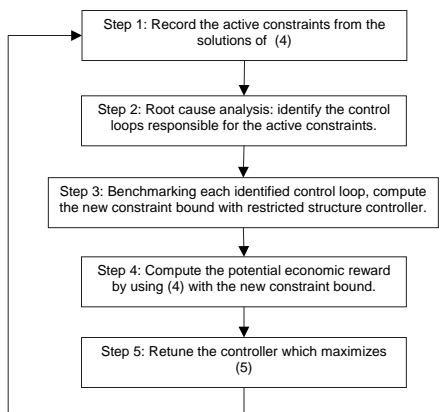

Figure 4: Controller benchmarking based on economic metrics

disturbance, see Figure. 5. The PID control system

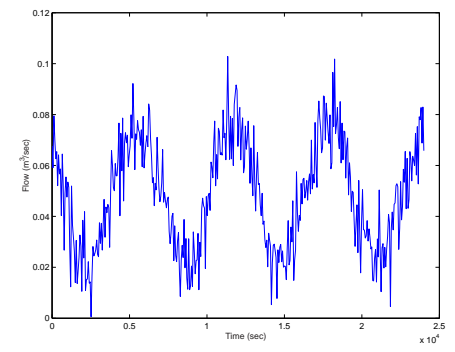

Figure 5: Input Flow into separator train

associated with each separator is tasked with keeping the level in the vessel constant. However, because of the sinusoidal nature of the input flow of reservoir fluid, the existing PID solution did not meet the requirements. The level alarm setting for both tanks is from $0.3 \mathrm{~m}(\min )$ to $1.95 \mathrm{~m}(\max )$. A Matlab / Simulink model of the first two stages of the separation system (high pressure (HP) and medium pressure (MP) separators) was developed and validated using the real plant data. The process characteristics were obtained by linearizing this model around the normal operating conditions and using balanced model reduction techniques[13]. One simulation with the original PID controller is shown in Figure. 6.

\subsection{Benchmarking Analysis}

First a careful analysis of the process revenue generation has been carried out. It is clear that the main process objective is to maximize production. Consequently a high process up time is the most important requirement, the estimated economic loss due to a trip/plant shutdown is about 500,000 pounds. The input flow oscillatory disturbance must be controlled and not transmitted down stream to other process units. It is also observed that the separation perfor-

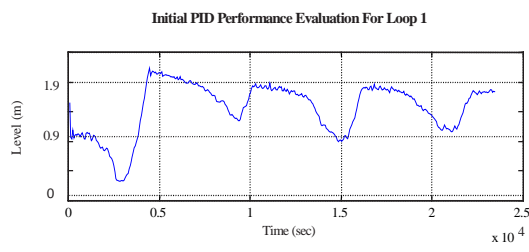

(a)

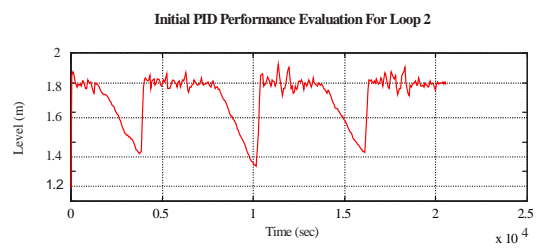

(b)

Figure 6: (a) Liquid level of HP separator (b) Liquid level of MP separator

mance is not sensitive to the liquid level. Based on the above analysis, an economic metric function for a whole month is formed as following:

$$
\begin{aligned}
& J_{\text {month }}=30 *\left(14000 * \sigma_{\text {flow }}^{2}+350 * \sigma_{\text {level }}^{2}\right) \\
& \text { s.t. } \quad 0.3 m \leq L_{l} \leq L \leq L_{h} \leq 1.95 m
\end{aligned}
$$

$\sigma_{\text {flow }}^{2}$ and $\sigma_{\text {level }}^{2}$ are the variances of the outflow and the tank level respectively, while $L_{l}$ and $L_{h}$ are the soft constraints of allowable low/upper liquid levels. The cost coefficients were obtained from steady-state simulations and estimates provided by plant operating staff.

Because of the sinusoidal nature of the input flow, it is impossible to have $\sigma_{\text {flow }}^{2}$ and $\sigma_{\text {level }}^{2}$ to be zero at the same time. In fact, keeping the flow constant and keeping the level constant are two conflicting requirements. The optimal solution of (6) reveals the following facts:

- The level in the slugcatcher must be kept within the physical limits to avoid plant shutdown.

- The minimum of (6) is obtained when $\sigma_{\text {flow }}^{2}$ is minimized.

- By relaxing soft constraints $L_{l}$ and $L_{h}$, the minimum of (6) can be further reduced.

In summary, the separators should be used as surge/buffer tanks. The control objective is to dampen the changes in output flow while keeping the liquid level in the vessel between limits. The original controller is trying to keep the tank level to the setpoint (i.e. $L_{l}$ and $L_{h}$ were set around the set-point.), 


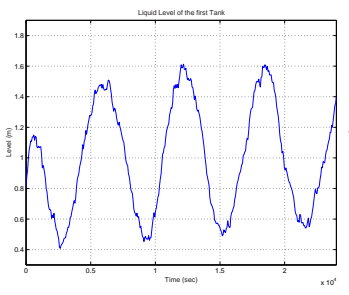

(a)

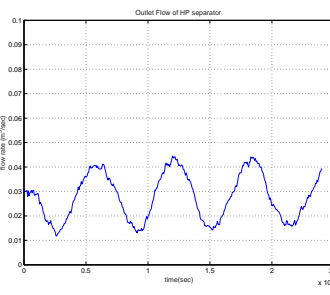

(b)
Figure 7: HP separator under proportional control

and as a result the tank is acting as a pipe with poor flow variation damping. This controller should be replaced by a so called average-level controller [12].

\subsection{Controller redesign based on economic met- rics}

The optimal level controller are in effect non-linear constrained optimizers, where the objective is to minimize the maximum rate of change of the outlet flow subject to the level remaining within bound [9]. Extra measurements of inflow, outlet rates are needed to implement such a nonlinear controller. If this new controller is to be installed, the plant has to be shut down to install new sensors and controllers. In this case, the term $J_{c}(y)$ in equation (5) is 500,000 pounds (equivalent to the cost of a shutdown). The economic loss is so big that it would outweigh the benefit of retuning the controller. Instead, we choose to detune the original PID controller into a proportional level controller [12]. The simulation result is shown in figure.7. It is clear that the tank level has been successfully regulated, the variance of flow is about a third of the original PID controller. Furthermore, the outlet flow is smooth.

\section{Conclusions}

In this paper, we present a controller benchmarking framework inspired by the practice of business benchmarking. The specific features of benchmarking activities are discussed. A guideline on how to choose benchmarking metrics is presented. Since benchmarking activity is a benefit-driven activity, we propose to use economic metrics directly for controller benchmarking. The process of debottlenecking provides us with the vital information on which parts of the process need to be further investigated. By integrating low level controller benchmarking techniques with the high level economic optimization, we make sure that the controller benchmarking would result in a better economic performance.

\section{References}

[1] K.J. Astrom. Introduction to stochastic control. Academic Press, London, 1971.

[2] L. Desborough and T. Harris. Performance assessment measures for univariate feedback control. The Canadian Journal of Chemical Engineering, Vol.70:pp.1186-1197, 1992.

[3] L. Desborough and T.J. Harris. Performance assessment measures for univariate feedforward/feedback control. The Canadian Journal of Chemical Engineering, Vol.71:pp.605-612, 1993.

[4] MJ Grimble. Restricted-structure LQG optimal control for continuous-time systems. IEE Proc. Control Theory Appl., Vol.147:pp.185195, 2000.

[5] MJ Grimble. Controller performance benchmarking and tuning using generalised minimum variance control. Automatica, Vol 38: pp.21112119, 2002.

[6] MJ Grimble. and D. Uduehi. Process control loop benchmarking and revenue optimization. American Control Conference, Arlington, Virginia, 2001.

[7] T.J. Harris. Assessment of control loop performance. The Canadian Journal of Chemical Engineering, Vol.67:pp.856-861, 1989.

[8] B. Huang, S.L. Shah, and E.K. Kwok. Good, bad or optimal? performance assessment of multivariable processes. Automatica, Vol.33:pp.1175$1183,1997$.

[9] A. Hugo. Process controller performance assessment. Hydrocarbon Processing, Vol.80:pp.8590, 2001.

[10] R. Miller and L. Desborough. Increasing customer value of industrial control performance monitoring - Honeywell's experience. Proceedings of Chemical Process Control VI, 2001.

[11] S.L. Shah, R. Patwardhan, and B. Huang. Multivariate controller performance analysis: methods, applications and challenges. Proceedings of Chemical Process Control VI, 2001.

[12] F.G.Shinskey. Feedback controllers for the process industries. McGraw-Hill, Inc., New York, 1994.

[13] D. Uduehi, D. Laing, A.W.Ordys and M.Grimble. Crude oil separation system optimisation using model based control. Process Control and Instrumentation 2000, Glasgow, Scotland. 